\title{
Severe Bone Marrow Suppression Associated with Use of Clopidogrel
}

\section{Zacharia $\mathrm{G}^{*}$, Randhawa $\mathrm{A}^{1}$, Marino $\mathrm{N}^{2}$ and Spaccavento $\mathrm{C}^{1}$}

${ }^{1}$ Division of Hematology and Oncology, Lenox Hill Hospital, New York, NY, USA

${ }^{2}$ Department of Cardiovascular Medicine, Lenox Hill Hospital, New York, NY, USA

*Corresponding author: Zacharia G, Division of Hematology and Oncology, Lenox Hill Hospital, 100 East 77 ${ }^{\text {th }}$ Street, New York, NY, USA 10075, Fax: 1212434 3413, Tel: 1212434 3124, E-mail: gezacharia@gmail. com

Citation: Zacharia G, Randhawa A, Marino N, Spaccavento C (2016) Severe Bone Marrow Suppression Associated with Use of Clopidogrel. J Case Rep Stud 4(4): 401. doi: 10.15744/2348-9820.4.401

Received Date: May 26, 2016 Accepted Date: August 27, 2016 Published Date: August 30, 2016

\begin{abstract}
A 60 year-old male presented with fever, malaise, and myalgias. The patient was admitted 3 weeks prior for acute coronary syndrome, underwent coronary stent placement, and was started on clopidogrel. He denied any chills, night sweats, chest or abdominal discomfort, or bleeding. He denied any history of alcohol, tobacco or illicit drug use and did not report any drug allergies.
\end{abstract}

Three weeks prior to admission, at the start of clopidogrel therapy, complete blood count was within normal limits. On admit, white blood cell count (WBC) was $0.9 \times 10^{\wedge} 3 / \mathrm{uL}$, hemoglobin (Hb) $10.9 \mathrm{~g} / \mathrm{dL}$, platelet count (PLT) 94,000/uL, absolute neutrophil count 0 , and reticulocyte percentage $0.8 \%$.

Extensive workup for infectious and rheumatologic etiologies was ultimately negative. He then underwent bone marrow biopsy which revealed a hypocellular marrow, relative erythroid hyperplasia, and marked myeloid hypoplasia. Megakaryocytes were slightly increased in number. There was no evidence of malignancy.

Clopidogrel was discontinued and he was supported with growth factor stimulating agents and empiric antibiotics. Blood counts recovered in 9 days and fevers subsided. On the day of discharge, WBC was 17.6, Hb 12.7, and PLT 121,000. He was discharged on prasugrel in lieu of clopidogrel and thereafter monitored closely. Six months later, blood counts returned to normal limits.

Keywords: Adverse Drug Event; Cardiology; Antiplatelet Agents

List of Abbreviations: WBC: White Blood Cell Count; Hb: Hemoglobin; PLT: Platelet Count; BUN: Blood Urea Nitrogen; AST: Aspartate Transaminase; ALT: Alanine Transaminase

\section{Introduction}

Clopidogrel is used extensively as an antiplatelet agent and it is of paramount importance to understand the safety profile of these agents. Some of the hematologic abnormalities seen with clopidogrel include agranulocytosis, bleeding, epistaxis, aplastic anemia and thrombotic thrombocytopenic purpura. Here, we present a case of severe pancytopenia caused by clopidogrel after coronary stent placement. Febrile pancytopenia is an extremely rare side effect of this drug and awareness is crucial in providing safe and effective patient care. It is important to stop the medication immediately and provide supportive care.

\section{Case report}

A 60 year-old male was admitted with fever, generalized malaise, myalgias and productive cough with whitish sputum production. The patient had been admitted approximately 3 weeks prior to the same hospital for acute coronary syndrome, underwent coronary stent placement, and was started on clopidogrel $75 \mathrm{mg}$ once daily, orally. He denied any chills, night sweats, chest or abdominal discomfort, hematuria, easy bruising or bleeding. There was no history of cytopenias in the past. He had a history of seizure episodes after coronary artery bypass graft in 1993 and was treated with phenytoin and phenobarbitol. Five years later, phenytoin was changed to topiramate. The patient's last seizure episode was more than 15 years ago and there was no recent change in his anti-seizure medications.

His past medical history was notable for coronary artery disease status-post percutaneous coronary intervention, coronary artery bypass graft, and seizure disorder. He denied any history of alcohol, tobacco or illicit drug use. He was not allergic to any medications.

On physical examination, his vital signs were as follows: temperature (maximum): 104.4 degrees Fahrenheit, blood pressure 133/62 mmHg, pulse 85 beats/minute, respiratory rate 20 breaths/minute, and oxygen saturation 97\% on room air. He was in no 
acute distress with no oropharyngeal lesions, no organomegaly, and no notable petechiae or ecchymosis. The rest of his physical examination was unremarkable.

Three weeks prior to admission, at the start of clopidogrel therapy, the patient's complete blood count showed a white blood cell count (WBC) $7.1 \times 10^{\wedge} 3 / \mathrm{uL}$; hemoglobin $(\mathrm{Hb}) 14.6 \mathrm{~g} / \mathrm{dL}$; platelet count (PLT) 151,000/uL.

On admission, the patient had a WBC $0.9 \times 10^{\wedge} 3 / \mathrm{uL} ; \mathrm{Hb} 10.9 \mathrm{~g} / \mathrm{dl}$; hematocrit 30.5\%; PLT 94,000/uL; absolute neutrophil count 0; mean corpuscular volume $87.1 \mathrm{fL}$; reticulocyte percentage $0.8 \%$; and a differential showing neutrophils $0 \%$, lymphocytes $96 \%$, and eosinophils $4 \%$. The patient's blood counts during the course of the admission are shown in Figure 1.
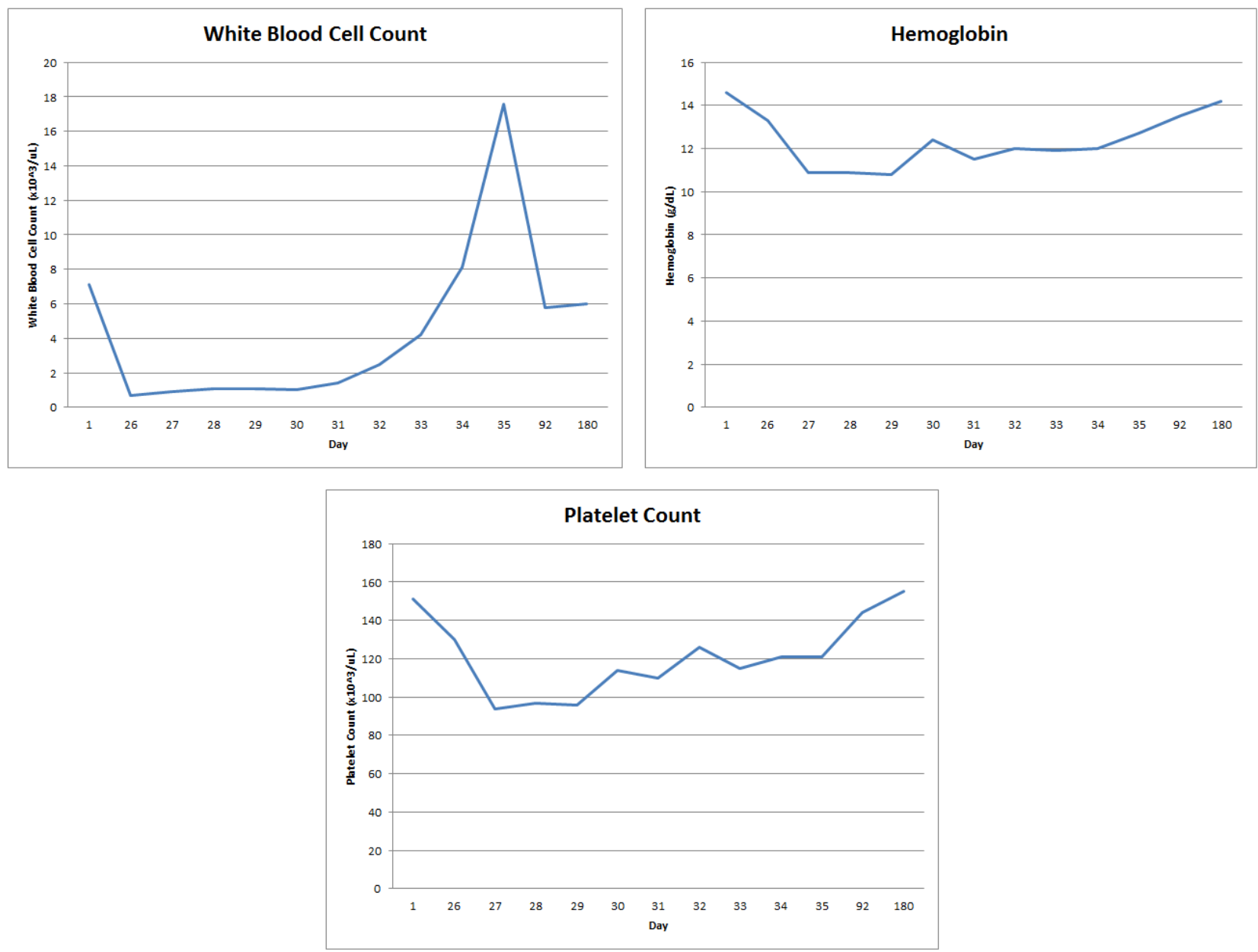

Figure 1: Complete Blood Count Trend: Day 1 - clopidogrel therapy initiated; Day 26 - patient admitted to the hospital; Day 35 hospital discharge; Days 92 \& 180 - post-hospital discharge follow-up labs. After the patient was admitted to the hospital on Day 26 of clopidogrel therapy, he was found to be pancytopenic and clopidogrel was discontinued. By hospital discharge and with further followup, pancytopenia had resolved and complete blood count values had returned to baseline

Other relevant laboratory data included: prothrombin time 16.8 seconds; partial thromboplastin time 31.5 seconds; BUN 16 mg/ dL; creatinine $1.02 \mathrm{mg} / \mathrm{dL}$; AST $47 \mathrm{U} / \mathrm{L}$; ALT $29 \mathrm{U} / \mathrm{L}$; total bilirubin $0.4 \mathrm{mg} / \mathrm{dL}$; total protein $5.9 \mathrm{~g} / \mathrm{dL}$; albumin $2.6 \mathrm{~g} / \mathrm{dL}$; and lactic acid $1.1 \mathrm{mmol} / \mathrm{L}$.

Blood and urine cultures were both negative. Chest radiograph showed no acute infiltrates. Influenza A and B antigens were negative. Serologies for hepatitis B and C, cytomegalovirus, parvovirus B19, Rocky Mountain spotted fever and typhus fever were also negative. Epstein-Barr virus serology showed previous exposure.

The peripheral blood smear showed slight anisocytosis, slight microcytosis, atypical reactive lymphocytes, and large platelets.

Bone marrow biopsy was done revealing a hypocellular marrow, relative erythroid hyperplasia, and marked myeloid hypoplasia with a myeloid-to-erythroid ratio of 0.7 to 1 . Maturation was seen in the erythroid series. Megakaryocytes were present and slightly increased in number (Figure 2). The increase in megakaryocytes and the large platelets seen on the peripheral smear was an indication perhaps of drug mediated immune destruction of the platelets. The hypocellular marrow with myeloid hypoplasia was indicative of bone marrow suppression. Flow cytometeric analysis revealed no evidence of acute leukemia or lymphoma, and cytogenetic studies showed a normal male karyotype. 


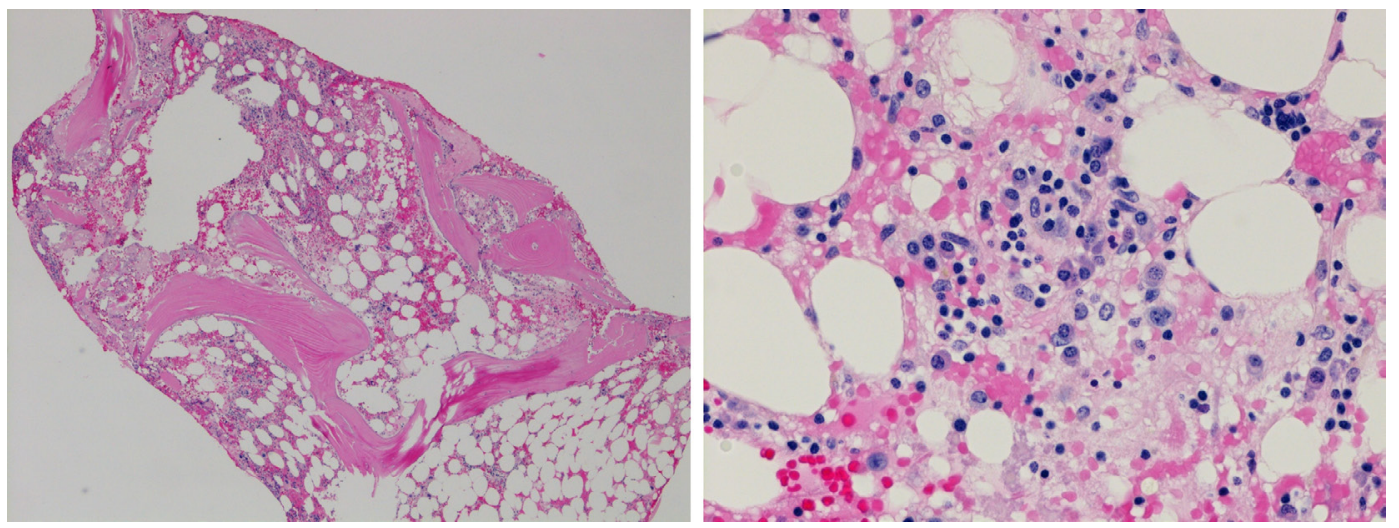

Figure 2: Bone marrow biopsy (4X; 40X Magnification). The bone marrow biopsy reveals a hypocellular marrow with marked myeloid hypoplasia and relative erythroid hyperplasia

Clopidogrel was discontinued on admission and the patient was supported with filgrastrim $300 \mathrm{mcg}$ subcutaneous daily and antibiotics for neutropenic fever. Antibiotics were started prophylactically and no source of infection was found. He was continued on his anti-seizure medications: topiramate and phenobarbitol. The blood counts recovered 9 days after discontinuation of clopidogrel, and fevers subsided as well. At that time, antibiotics and filgrastim were discontinued. The patient was discharged the following day with complete blood counts showing resolution of neutropenia and a WBC 17.6 x 10^3/uL; Hb $12.7 \mathrm{~g} / \mathrm{dL}$; PLT $121,000 \mathrm{u} / \mathrm{L}$.

He was discharged on prasugrel in lieu of clopidogrel and was thereafter monitored closely by his primary care physician and cardiologist. Six months later patient was admitted to our hospital for an unrelated cause and remained on prasugrel with normal complete blood counts showing WBC 6.0 x 10^3/uL; Hb $14.2 \mathrm{~g} / \mathrm{dL}$; mean corpuscular volume $85.7 \mathrm{fL}$; and PLT 155,000 u/L.

\section{Discussion}

Clopidogrel and ticlopidine are thienopyridine derivatives that inhibit platelet aggregation. Ticlopidine is extensively used to reduce the risk of sub-acute thrombosis after stent implantation. It has serious but rare side effects such as neutropenia, renal failure, thrombotic thrombocytopenic purpura, and bone marrow aplasia $[1,2]$.

These drugs reduce the overall rate of thromboembolic events in patients with atherosclerotic vascular disease after stent implantation. Ticlopidine is a first-generation thienopyridine. The use of ticlopidine has two major limitations, which are its safety profile and its inability to induce platelet inhibition rapidly.

Thus, clopidogrel, a second-generation thienopyridine, has largely replaced ticlopidine. Clopidogrel therapy may also be accompanied by rare life-threatening side effects. Clopidogrel may be found to be associated with severe bone marrow suppression manifested as bone marrow failure, aplastic anemia, thrombocytopenia, and neutropenia [2-4]. We present a case of severe pancytopenia caused by clopidogrel.

The incidence of thrombocytopenia and neutropenia induced by clopidogrel was reported to be $0.26 \%$ and $0.10 \%$, respectively in the CAPRIE (Clopidogrel versus aspirin in patients at risk of ischemic events) study [5]. The mechanism of aplastic anemia induced by clopidogrel is not well understood (possibilities include cumulative toxicity or idiosyncratic reaction). In a previous report, it was suggested that aplastic anemia occurred within 5 - 11 months of starting clopidogrel [4]. In our case, pancytopenia occurred within 3 weeks. In the literature, there are only a few reported cases of hematologic abnormalities due to clopidogrel, including rare cases of neutropenia $(0.1 \%)$, thrombocytopenia $(0.2 \%)$, one case of silent aplastic anemia, and one case of febrile pancytopenia [2-4].

As a result, clopidogrel may be a potential cause of pancytopenia irrespective of the duration of treatment, though rarely seen. Therefore, careful clinical and hematological monitoring should be carried out in the course of treatment with clopidogrel.

We propose that treatment with the drug be halted immediately if there is suspicion for any blood dyscrasias. In our patient, the alternative treatment with prasugrel was effective. Patients also require careful monitoring of their blood counts by their cardiologist or hematologist and abnormal blood counts should be investigated thoroughly.

\section{Conclusion}

Clopidogrel is used extensively as an antiplatelet agent and it is vital to understand the safety profile of these drugs. Some of the hematologic abnormalities seen with clopidogrel include bleeding, agranulocytosis, aplastic anemia and thrombotic thrombocytopenic purpura (TTP). Febrile pancytopenia is an extremely rare side effect of clopidogrel and awareness is crucial in providing safe and effective patient care. 


\section{References}

1. Love BB, Biller J, Gent M (1998) Adverse haematological effects of ticlopidine. Prevention, recognition and management. Drug Saf 19: 89-98.

2. McCarthy MW, Kockler DR (2003) Clopidogrel-associated Leukopenia. Ann Pharmacother 37: 216-9.

3. Andres E, Perrin AE, Alt M, Goichot B, Schlienger JL (2001) Febrile pancytopenia associated with clopidogrel. Arch Intern Med $161: 125$.

4. Trivier JM, Caron J, Mahieu M, Cambier N, Rose C (2001) Fatal aplastic anaemia associated with clopidogrel. Lancet 357 : 446.

5. CAPRIE Steering Committee (1996) A randomised, blinded, trial of clopidogrel versus aspirin in patients at risk of ischaemic events (CAPRIE). CAPRIE Steering Committee. Lancet 348: 1329-39.

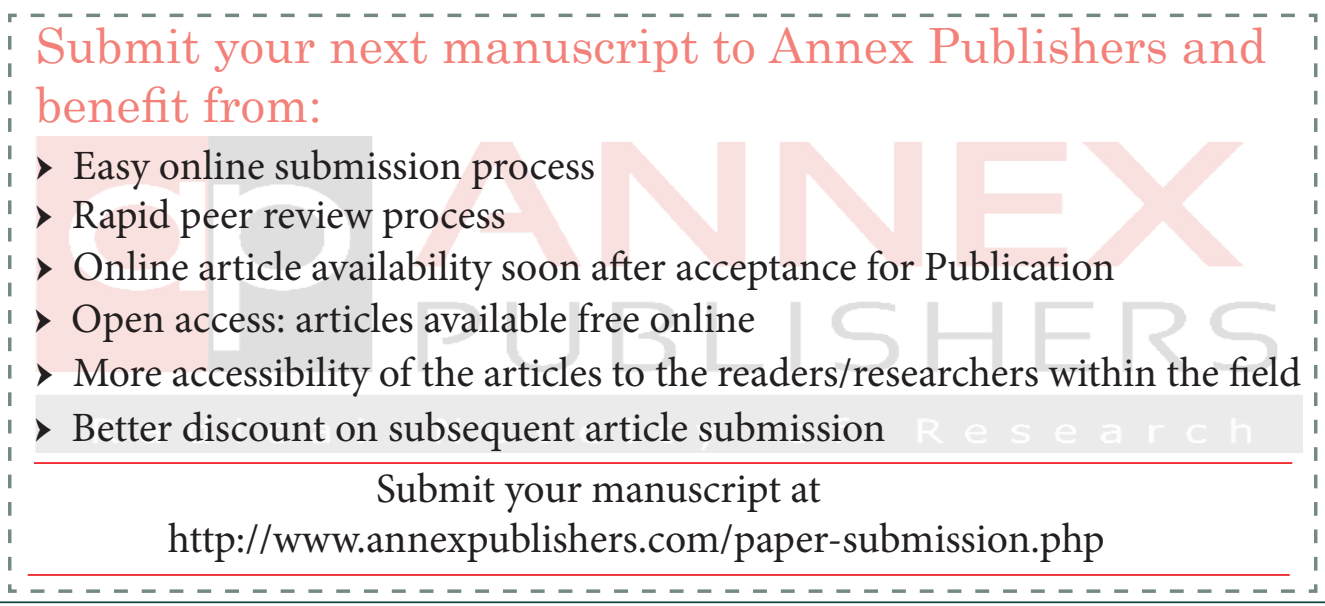

